\title{
Leucine-Rich Glioma-Inactivated Protein 1 Antibody-Positive Polyradiculopathy Associated with Epstein-Barr Virus Infection
}

\author{
Berrin Pelit Uzunalimoğlu ${ }^{a} \quad$ Abdülhamit Sağlam $^{a} \quad$ Büşra Şişman ${ }^{a}$ \\ Sefer Günaydın ${ }^{a}$ Esen Gül Uzuner ${ }^{b}$ Fikret Aysal ${ }^{c} \quad$ Erdem Tüzün $^{d}$ \\ Birgül Baştan ${ }^{a}$ \\ aDepartment of Neurology, Haseki Research and Training Hospital, Health Sciences \\ University, Istanbul, Turkey; ${ }^{b}$ Department of Pathology, Haseki Research and Training \\ Hospital, Health Sciences University, Istanbul, Turkey; 'Department of Neurology, Bakirkoy \\ Research and Training Hospital for Psychiatric and Neurological Diseases, Istanbul, Turkey; \\ dDepartment of Neuroscience, Aziz Sancar Institute for Experimental Medical Research, \\ Istanbul University, Istanbul, Turkey
}

\section{Keywords}

Polyradiculopathy · Leucine-rich glioma-inactivated protein 1 - Epstein-Barr virus . Antibody · Autoimmunity

\begin{abstract}
Epstein-Barr virus (EBV) has been associated with a plethora of neurological manifestations including polyneuropathy and polyradiculopathy. A 27-year-old man with a recent upper respiratory system infection presented with difficulty in walking. His neurological examination revealed reduced muscle strength in both proximal and distal lower limb muscles without sensory and autonomic signs. Needle electromyography showed abnormal spontaneous activity and reduced recruitment of motor units in muscles innervated by multiple lumbo-sacral roots. Cerebrospinal examination showed increased protein levels with normal cell counts. While spinal MRI was normal, whole-body $\mathrm{CT}$ and PET examination showed disseminated lymph node enlargement. Anti-EBV viral capsid antigen and anti-nuclear antigen IgG but not IgM was positive, whereas EBV PCR was negative in blood. Analysis of inguinal lymph node biopsy showed reactive lymphoid hyperplasia and EBV DNA. Leucine-rich glioma-inactivated protein 1 (LGI1) antibody was found in serum but not in CSF. All clinical, imaging, and electrophysiological findings improved following steroid and intravenous immunoglobulin treatment. These findings suggested the acute involvement of lumbo-sacral spinal roots and/or motor neurons. Purely motor polyradiculopathy has been reported in both EBV-positive and LGI1 antibody-positive patients, and EBV infection is known to precede different autoimmune
\end{abstract}


manifestations. Whether EBV infection may trigger LGI1 autoimmunity and cause involvement of spinal motor roots and/or motor neurons needs to be further studied.

\author{
(C) 2021 The Author(s). \\ Published by S. Karger AG, Base
}

\title{
Introduction
}

Neurological manifestations of Epstein-Barr virus (EBV) include meningitis, encephalitis, myelitis, and peripheral nerve manifestations including mononeuropathy, polyneuropathy, and polyradiculopathy. Some patients with polyradiculopathy may present with purely motor symptoms implying isolated involvement of anterior nerve roots and motor neurons [1]. Mechanisms by which EBV causes peripheral nerve manifestations are not precisely understood. We have encountered a patient presenting with findings that are compatible with lumbo-sacral spinal root and/or motor neuron involvement, recent EBV infection, and antineuronal autoimmunity, suggesting that autoimmune mechanisms might partake in postviral peripheral nerve/motor neuron manifestations.

\section{Case Report}

A 27-year-old man presented with 2 weeks of difficulty in walking. One month prior to neurological symptom onset, he had developed fever, general malaise, sore throat, postnasal drip, and cervical lymphadenopathy. Otherwise, his past medical and family history was unremarkable. Upper respiratory system symptoms had ceased 1 week before the onset of neurological symptoms, the pharynx looked normal, but the spleen was slightly enlarged. Neurological examination showed reduced muscle strength in both proximal (medical research council [MRC] 2-3/5) and distal (MRC 3-5/5) lower limb muscles. Deep tendon reflexes of lower limbs could not be elicited. There were no sensory deficits. Electromyography performed 1 week after the onset of neurological symptoms showed normal motor and sensory nerve conduction and tibial nerve F-responses. Needle electromyography of lower limb muscles (plantar flexors and dorsiflexors, knee extensors and flexors, hip flexors, extensors, and abductors) showed increased insertional activity and abnormal spontaneous activity at rest. Electromyography also showed reduced recruitment of polyphasic motor units with high amplitude and long duration in muscles innervated by multiple lumbo-sacral root distribution bilaterally. No pathological features were noted in trunk and upper limb muscles. Potential infectious, autoimmune, and neoplastic etiologies were investigated.

Contrast-enhanced cranial and spinal MRIs were normal, and no spinal nerve root compression was observed. Cerebrospinal fluid (CSF) examination revealed increased protein (129 mg/dL), normal glucose concentration, and no lymphocytes. Fungal and bacterial (including tuberculosis) cultures of CSF proved negative. There were no CSF-specific oligoclonal bands, IgG index was normal, and there were no atypical cells in CSF.

Blood biochemistry, total blood count, and serum/urine immune electrophoresis were normal, and peripheral blood smear did not reveal atypical cells. Serological tests for HBV, HCV, HSV, syphilis, HIV, COVID-19, Brucella, and CMV were negative, whereas anti-EBV viral capsid antigen (VCA) and anti-nuclear antigen (EBNA) IgGs were positive. However, anti-VCA and EBNA IgM were not found in serum, and EBV PCR (RealStar EBV PCR Kit 1.0; Altona Diagnostics $\mathrm{GmbH}$, Hamburg, Germany) was negative in blood, suggesting past EBV infection. Due

\section{Karger'}


Fig. 1. Serum antibodies of the patient react with HEK293 cells expressing LGI1 (green fluorescence). Original magnification, ×20. HEK293, human embryonic kidney 293; LGI1, leucine-rich glioma-inactivated protein 1.

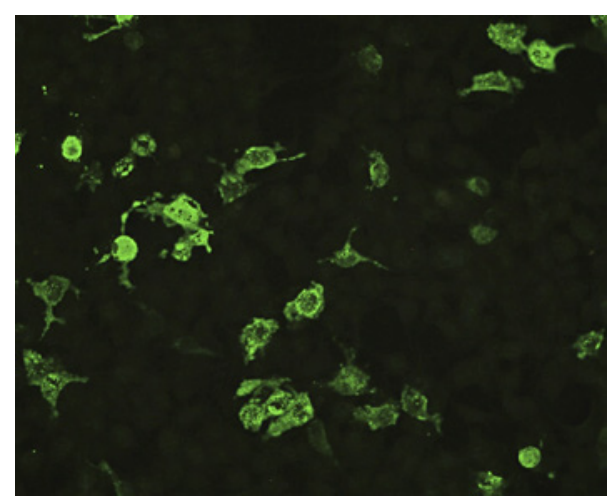

to absence of evidence for acute EBV infection, EBV PCR was not done with CSF. Also, since past EBV infection is highly common in healthy population, autoimmune and paraneoplastic etiologies were investigated.

Antibody panels for gangliosides (anti-GM1, GM2, GM3, GD1a, GD1b, GT1b, GQ1b IgG, and $\operatorname{IgM}$ ) and vasculitic and paraneoplastic disorders were all negative. While a panel for autoimmune encephalitis-related antibodies proved negative for serum/CSF N-methylD-aspartate receptor (NMDAR), AMPA receptor, contactin-associated protein-like 2 (CASPR2), and $\mathrm{GABA}_{\mathrm{B}}$-receptor-antibodies, leucine-rich glioma-inactivated protein 1 (LGI1) antibody was found in serum (1:800 dilution) but not in CSF (Euroimmun, Luebeck, Germany) (Fig. 1). While whole-body CT showed enlarged contrast-enhancing para-aortic and inguinal lymph nodes, whole-body PET showed increased ${ }^{18}$ F-fluoro-2-deoxy-D-glucose uptake in the spleen, bilateral lymph nodes of the neck, submandibular region, thorax, abdomen, and inguinal region (Fig. 2), raising suspicion for lymphoma.

Analysis of the inguinal lymph node biopsy specimen using antibodies to CD3, CD20, Bcl2, Bcl6, CD10, and Ki67 did not show identical clonal T or B-cell population but reactive lymphoid hyperplasia indicative of viral infection. EBV DNA was demonstrated in lymph node tissue by PCR. These findings suggested involvement of lumbo-sacral spinal roots and/or motor neurons as postviral manifestation of a recent primary EBV infection. Muscle weakness showed remarkable amelioration (MRC 4-5/5) following pulse methylprednisolone, intravenous immunoglobulin, and subsequently administered oral methylprednisolone for 2 months. At the 4-month follow-up visit, neurological examination and electromyography investigation were normal. The whole-body CT investigation was also normal, and no enlarged lymph nodes or contrast enhancement was noted.

\section{Discussion}

Acute polyradiculopathy may occasionally present with purely motor symptoms without sensory and autonomic manifestations, as in our case [2]. Although lymphoma was seriously considered, normal CSF and lymph node pathological examination and regression of enlarged lymph nodes during follow-up brought forward the postviral autoimmune mechanisms as the more likely pathogenic factor. Similar extensive involvement of lymph nodes has been shown in other EBV+ patients without lymphoma [3]. Purely motor polyradiculopathy has been reported in primary EBV infections with the coined terms of poliomyelitis-like and anterior horn syndrome [1]. Clinical and electromyography findings of our patient are congruent with both spinal motor root and motor neuron involvement, and there is no evidence to differentiate whether the lesions are in spinal roots, motor neurons, or both. Thus, we have used the

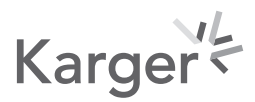


Fig. 2. Multiple focal areas of increased 18F-fluoro-2-deoxy-D-glucose uptake were seen in the bilateral lymph nodes of cervical, thoracic, abdominal, and inguinal regions in the positron emission tomography scan.

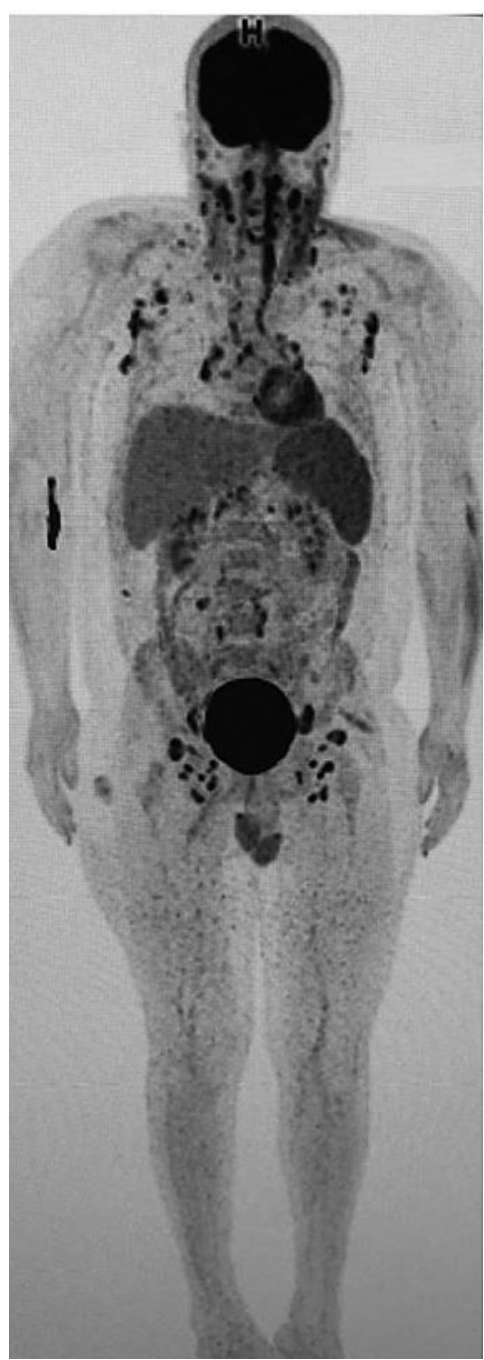

term polyradiculopathy as a term to indicate the clinical and electrophysiological syndrome of the patient [2], which might have been caused by spinal root and/or motor neuron involvement. A potential mechanism for this combination of findings may be selective invasion of spinal motor roots or motor neurons by EBV. However, to our knowledge, there is no substantial published evidence for this mechanism in the literature.

Anti-LGI1 seropositivity, found in our study, has implied autoimmune etiology as an alternative cause. Although LGI1 antibodies were only found in the serum of our case, absence or lower titers of anti-neuronal antibodies in CSF and normal IgG index are quite common findings in LGI1 autoimmunity and suggest peripherally initiated autoimmune response [4]. Nevertheless, in CSF LGI1 antibody-negative patients, intrathecal immunoglobulin repertoire expansion can be detected by B-cell immune repertoire sequencing, suggesting intrathecal B-cell activation in LGI1 autoimmunity [5]. Also, the reasonably high titer of serum LGI1 antibody and absence of IgG reactivity with cells transfected with other antigens indicated that our antibody finding was not due to nonspecific IgG reactivity.

Antibodies to voltage-gated channel (VGKC) complex subunits CASPR2 and LGI1 were found in patients with polyneuropathy and neuropathic pain. Cases with motor neuron disorder-like presentations may also display anti-CASPR2 [6, 7]. Moreover, mutations of certain VGKC subunits may lead to motor neuron dysfunction, and antibodies to the VGKC 
complex are found in patients with motor neuron disorders [8, 9], implying the significance of the VGKC complex in anterior horn functions.

EBV and HSV, both members of the Herpesviridae family, are known to precede and possibly facilitate NMDAR autoimmunity [10]. To our knowledge, our case is the first example of anti-LGI1 and EBV temporal association in a patient with central or peripheral nervous system manifestations. Our results imply that LGI1 autoimmunity may act as an auxiliary factor for motor nerve and/or motor neuron impairment and autoantibodies might be involved in physiopathology of EBV-associated neurological disorders.

\section{Statement of Ethics}

The study is exempt from ethics committee approval since it is a case report. Written informed consent was obtained from the patient for publication of this case report and any accompanying images.

\section{Conflict of Interest Statement}

The authors have no conflicts of interest to declare.

\section{Funding Sources}

This research did not receive any specific grant from funding agencies in the public, commercial, or not-for-profit sectors. All medical investigations and laboratory tests were done as a part of routine differential diagnosis.

\section{Author Contributions}

Acquisition, analysis, and interpretation of data for the work were done by Berrin Pelit Uzunalimoğlu, Abdülhamit Sağlam, Büşra Şişman, Sefer Günaydın, Esen Gül Uzuner, Fikret Aysal, and Birgül Baștan. Drafting the work and revising it critically for important intellectual content were done by Berrin Pelit Uzunalimoğlu, Fikret Aysal, Erdem Tüzün, and Birgül Baştan. Final approval of the version to be published was provided by all authors.

\section{Data Availability Statement}

Research data are not publicly available on legal and ethical grounds.

\section{References}

1 Poorthuis MHF, Battjes S, Dorigo-Zetsma JW, de Kruijk JR. Primary Epstein-Barr virus infection in immunocompetent patients with acute transverse myelitis and a combination of polyradiculitis and anterior horn syndrome as neurological manifestations. BMJ Case Rep. 2018;2018:bcr2018225333.

2 McGonagle TK, Levine SR, Donofrio PD, Albers JW. Spectrum of patients with EMG features of polyradiculopathy without neuropathy. Muscle Nerve. 1990;13(1):63-9.

3 Lustberg MB, Aras O, Meisenberg BR. FDG PET/CT findings in acute adult mononucleosis mimicking malignant lymphoma. Eur J Haematol. 2008;81(2):154-6. 
4 Irani SR, Stagg CJ, Schott JM, Rosenthal CR, Schneider SA, Pettingill P, et al. Faciobrachial dystonic seizures: the influence of immunotherapy on seizure control and prevention of cognitive impairment in a broadening phenotype. Brain. 2013;136(Pt 10):3151-62.

5 Lehmann-Horn K, Irani SR, Wang S, Palanichamy A, Jahn S, Greenfield AL, et al. Intrathecal B-cell activation in LGI1 antibody encephalitis. Neurol Neuroimmunol Neuroinflamm. 2020;7(2):e669.

6 Lancaster E, Huijbers MG, Bar V, Boronat A, Wong A, Martinez-Hernandez E, et al. Investigations of caspr2, an autoantigen of encephalitis and neuromyotonia. Ann Neurol. 2011;69(2):303-11.

7 Gadoth A, Pittock SJ, Dubey D, McKeon A, Britton JW, Schmeling JE, et al. Expanded phenotypes and outcomes among 256 LGI1/CASPR2-IgG-positive patients. Ann Neurol. 2017;82(1):79-92.

8 Khare S, Nick JA, Zhang Y, Galeano K, Butler B, Khoshbouei H, et al. A KCNC3 mutation causes a neurodevelopmental, non-progressive SCA13 subtype associated with dominant negative effects and aberrant EGFR trafficking. PLoS One. 2017;12(5):e0173565.

9 Godani M, Zoccarato M, Beronio A, Zuliani L, Benedetti L, Giometto B, et al. Voltage-gated potassium channel antibodies in slow-progression motor neuron disease. Neurodegener Dis. 2017;17(1):59-62.

10 Linnoila JJ, Binnicker MJ, Majed M, Klein CJ, McKeon A. CSF herpes virus and autoantibody profiles in the evaluation of encephalitis. Neurol Neuroimmunol Neuroinflamm. 2016;3(4):e245. 